\title{
Unified Theory of Force Fields (Electromagnetic and Gravitational)
}

\author{
Chaykin Andrey \\ Home, Moscow, Russia \\ Email: 9060728184@mail.ru, andreychaykin2016@gmail.com
}

How to cite this paper: Andrey, C. (2017) Unified Theory of Force Fields (Electromagnetic and Gravitational). World Journal of Condensed Matter Physics, 7, 31-35. https://doi.org/10.4236/wjcmp.2017.71003

Received: November 26, 2016

Accepted: February 25, 2017

Published: February 28, 2017

Copyright $\odot 2017$ by author and Scientific Research Publishing Inc. This work is licensed under the Creative Commons Attribution International License (CC BY 4.0).

http://creativecommons.org/licenses/by/4.0/

\begin{abstract}
In this paper, the superfluid substance is described by the same equations of the electromagnetic field and the gravitational field. The gravitational mass is sufficiently considered as the gravitational charge, having the same dimensions as electric charge.
\end{abstract}

\section{Keywords}

Gravity, Electromagnetic Field, Maxwell's Equations, Helium II, Gravitodynamics

\section{Introduction}

Leibniz imagined the space was filled initially with liquid matter. Taking a superfluid substance filling all space as model I explain electromagnetism over a stream superfluid substance: Electrical phenomena-progressive (potential), magnetic properties-vortex (solenoidal). For an explanation of gravitation tubular threads in a superfluid substance is formed. The density thread is the gravity potential. Electromagnetic waves are caused by disturbances of the electromagnetic field and produced by the accelerated motion of charged particles, and gravitational waves are caused by the same accelerated motion of mass (the gravitational charge). Gravitational waves are generated by accelerated motion of gravitational charges (masses) [1].

The gravitational field and the electromagnetic field obey the equations of Maxwell-Chaykin.

These equations are fixed by the author of the differential equations of Maxwell:

$$
\nabla \cdot \boldsymbol{E}=\operatorname{div} \boldsymbol{E}=4 \pi \rho-\frac{1}{C} \cdot \frac{\partial|\boldsymbol{B}|}{\partial t}
$$




$$
\begin{gathered}
\nabla \times \boldsymbol{E}=\operatorname{rot} \boldsymbol{E}=0 \\
\nabla \times \boldsymbol{B}=\operatorname{rot} \boldsymbol{B}=\frac{4 \pi}{C} \cdot \boldsymbol{J}+\frac{1}{C} \cdot \frac{\partial \boldsymbol{E}}{\partial t} \\
\nabla \cdot \boldsymbol{B}=\operatorname{div} \boldsymbol{B}=0
\end{gathered}
$$

\subsection{Electrodynamics}

1) Except for vortex electric field $\operatorname{rot} \boldsymbol{E}=0$, the electric field is always potentially.

2) Time-varying magnetic field is interpreted as a density charge displacement $\rho_{\text {dis }}=\frac{1}{C} \cdot \frac{\partial|\boldsymbol{B}|}{\partial t}$ and summed up with the density of real charge.

\subsection{Gravitodynamics}

1) Gravitational mass is considered as the gravitational charge, which has the same dimensions as electric charge.

$$
q_{g r}=\sqrt{G} m
$$

2) Gravity currents are conventional mechanical impulses. It leads to a vortex gravitational field, like in electrodynamics and with the same consequences.

If we consider gravity currents and charges displacement in the Poisson equation, it eliminates the need to bend space and retreat from Euclidean space to describe the gravitational field [2] [3].

\section{Theory}

\subsection{Electromagnetic Field}

There is a substance filling all space. The property of superfluidity ensures the equality of the inertial frame and the invariance of charge. The two-fluid model substance (similar to the He II (helium)) provides mutual counter-movement of the two components without visible real flow of the whole fluid. This substance is superfluid liquid having density $\boldsymbol{\rho}_{H}$ and velocity field $\boldsymbol{V}_{H}$ (H-in honor of Gelmgoltsa).

$$
\boldsymbol{V}_{H}=\boldsymbol{V}_{p}+\boldsymbol{V}_{v}
$$

$\boldsymbol{V}_{p}$ - potential velocity field,

$$
\operatorname{rot} V_{p}=0
$$

$\boldsymbol{V}_{v}$ - vortex (solenoidal) velocity field,

$$
\operatorname{div} \boldsymbol{V}_{v}=0
$$

Taken as the postulate, for the electric and magnetic fields:

$\boldsymbol{E}=\sqrt{\rho_{H}} \cdot \boldsymbol{V}_{p}$-intensity of the electric field.

$\boldsymbol{B}=\sqrt{\rho_{H}} \cdot \boldsymbol{V}_{v}$-intensity of the magnetic field.

The equations of electrodynamics we rewrite in the form:

$$
\operatorname{div} \boldsymbol{E}=4 \pi \rho-\frac{1}{C} \cdot \frac{\partial|\boldsymbol{B}|}{\partial t}
$$




$$
\begin{gathered}
\operatorname{rot} \boldsymbol{E}=0 \\
\operatorname{rot} \boldsymbol{B}=\frac{4 \pi}{C} \cdot \boldsymbol{J}+\frac{1}{C} \cdot \frac{\partial \boldsymbol{E}}{\partial t} \\
\operatorname{div} \boldsymbol{B}=0
\end{gathered}
$$

$|\boldsymbol{B}|$-absolute intensity of the magnetic field.

From these corrected Maxwell equations follows:

1) Electric field $\boldsymbol{E}$ is always potential.

2) The electromagnetic wave has a longitudinal component $\boldsymbol{E}$ with the properties of the particles and the transverse component $\boldsymbol{B}$-the essence of the wave.

3) The main stream of electromagnetic radiation is directed via $\boldsymbol{E}$, and the Poynting vector $\boldsymbol{E} \times \boldsymbol{B}$-side stream is responsible for a divergence of the ray.

4) The equation Faraday's Electromagnetic induction is valid only for non mono connected areas and in differential form does not make sense.

5) Accelerators type Bevatron, Foucault currents and other effects allegedly caused by the vortex field $\boldsymbol{E}$, can be explained with the help of, what I call, the charge density of displacement: $\rho_{\mathrm{dis}}=\frac{1}{C} \cdot \frac{\partial|\boldsymbol{B}|}{\partial t}$

6) Magnetic field-an independent entity, is not an effect of "second order" of the electric field.

In summary, conclude:

1) The carrier of the electromagnetic field is a superfluid liquid (it can be paired to bosons neutrinos or something what we do not known);

2) The singular regions, the points (drain, source) have the properties of electric charge;

3) The property of superfluidity ensures the equality of inertial reference systems and invariance of charge;

4) The two-fluid model substance (similar to the Helium II) provides mutual counter-movement of the two components without visible real flow of the whole fluid.

\subsection{Gravitational Field}

Gravitational field, initially, according to Newton and Hooke and others, is the force field.

The trajectory of the gravitating masses should be perpendicular (normal) to the equipotential surfaces. These surfaces are determined by the distribution of other gravitating masses (charges) and currents (mechanical impulses).

To equate gravity with the forces of inertia, with the forces Coriolis, that is, with forces unable to produce work for the true physics is unacceptable [4].

Gravitating mass does not curve space. They spin liquid substance between them (it is energetically profitable for the liquid). In a superfluid substance, like in rotating He II (helium), the formation of a tubular filament. The gradient of the linear density $\rho_{\mathrm{L}}$, these tubular filaments, are manifested as the gravitational field intensity $\boldsymbol{E}_{g r}$ (potential). 


$$
\boldsymbol{E}_{g r}=\sqrt{G} \cdot \operatorname{grad} \boldsymbol{\rho}_{\mathrm{L}}
$$

$G$-gravitational constant of Newton.

The density of gravitational charge $\rho_{g r}$ :

$$
\rho_{g r}=\sqrt{G} \cdot \operatorname{div} \operatorname{grad} \rho_{\mathrm{L}}=\Delta \rho_{\mathrm{L}} \cdot \sqrt{G}
$$

$\triangle$-Laplace operator.

\subsection{Gravitational Charge $q_{g r}$}

$$
\boldsymbol{q}_{g r}=\sqrt{G} \cdot m
$$

$m-$ mass (in grams)

Thus, the ratio $\sqrt{G}$ gives the gravitational mass of the $\boldsymbol{q}_{g r}$ the same dimension as that of the electric charge $\boldsymbol{q}$, in CGS units [ $\mathrm{g} 1 / 2, \mathrm{~cm} 3 / 2, \mathrm{sec}-1]$. And as a consequence of intensity and currents the gravitational field has the appropriate dimension, as in electrodynamics.

Dimension in the CGS $(\mathrm{cm}, \mathrm{g}, \mathrm{sec}) \boldsymbol{E}_{g r}$ and $\boldsymbol{\rho}_{g r}$ is the same as in electrodynamics. The laws of electrodynamics are valid for gravity [5].

Let's us assume that the equations for gravitodynamics:

$$
\begin{aligned}
\operatorname{div} \boldsymbol{E}_{g r}= & 4 \pi \boldsymbol{\rho}_{g r}-\frac{1}{C_{g r}} \cdot \frac{\partial\left|\boldsymbol{B}_{g r}\right|}{\partial t} \\
& \operatorname{rot} \boldsymbol{E}_{g r} \equiv 0 \\
\operatorname{rot} \boldsymbol{B}_{g r}= & \frac{4 \pi}{C_{g r}} \cdot \boldsymbol{J}_{g r}+\frac{1}{C_{g r}} \cdot \frac{\partial \boldsymbol{E}_{g r}}{\partial t} \\
& \operatorname{div} \boldsymbol{B}_{g r} \equiv 0
\end{aligned}
$$

$\rho_{g r}$-density of the gravitational charge,

$\boldsymbol{B}_{g r}$-intensity of the vortex gravitational field,

$\boldsymbol{E}_{g r}$-intensity potential of the gravitational field,

$\boldsymbol{J}_{g r}$ - density of the gravity current (the density of the kinetic impulse),

$C_{g r}$-speed of gravitational waves.

From these equations it follows:

1) The precession of mercury's orbit is calculated in the same way as the motion of a charged particle in the Coulomb field (see Landau, Lifshitz "Theory of fields").

2) Gravitational currents different directed-push off, one direction-attracted.

\section{Conclusion}

In the end, the UNIFIED THEORY of FORCE FIELDS is that the General equation of any strong ELECTROMAGNETIC or GRAVITATIONAL fields has the form, and can be calculated by equations of Maxwell's-Chaykin:

$$
\begin{gathered}
\operatorname{div} \boldsymbol{E}=4 \pi \rho-\frac{1}{C} \cdot \frac{\partial|\boldsymbol{B}|}{\partial t} \\
\operatorname{rot} \boldsymbol{E}=0
\end{gathered}
$$




$$
\begin{gathered}
\operatorname{rot} \boldsymbol{B}=\frac{4 \pi}{C} \cdot \boldsymbol{J}+\frac{1}{C} \cdot \frac{\partial \boldsymbol{E}}{\partial t} \\
\operatorname{div} \boldsymbol{B}=0
\end{gathered}
$$

The laws of electrodynamics are valid for gravity, but have different interpretations.

\section{References}

[1] Klein, M. (1984) Mathematics: The Loss of Certainty. Mir, Moscow.

[2] Davis, P. (1985) A Random Universe. Nauka, Moscow.

[3] Novikov, I.D. (1983) The Evolution of The Universe. Nauka, Moscow.

[4] Rashevsky, P.K. (1967) Riemannian Geometry and Tensor Analysis. Nauka, Moscow.

[5] Boyarkin, O.M. (2006) Introduction to Physics of Elementary Particles. Nauka, Moscow.

Submit or recommend next manuscript to SCIRP and we will provide best service for you:

Accepting pre-submission inquiries through Email, Facebook, LinkedIn, Twitter, etc. A wide selection of journals (inclusive of 9 subjects, more than 200 journals)

Providing 24-hour high-quality service

User-friendly online submission system

Fair and swift peer-review system

Efficient typesetting and proofreading procedure

Display of the result of downloads and visits, as well as the number of cited articles Maximum dissemination of your research work

Submit your manuscript at: http://papersubmission.scirp.org/

Or contact wjcmp@scirp.org 